\title{
Non-Singular Homogeneous Polyhedral Heat Cloak and Its Realization
}

\author{
Qingxiang Ji, ${ }^{1,2}$ Xueyan Chen, ${ }^{1,2}$ Jun Liang, ${ }^{3}$ Vincent Laude, ${ }^{2}$ Sébastien Guenneau, ${ }^{4}$ Guodong Fang ${ }^{1 *}$ and Muamer Kadic ${ }^{2}$
}

As most arbitrarily shaped cloaks can be approximated by polyhedra and further divided into a series of tetrahedra, we propose in this paper a linear mapping approach to design cloaks with tetrahedron shapes (i.e. tetrahedral cloaks). Homogeneous material properties of the cloak are straightforwardly obtained from coordinates of typical points. Consequently, most arbitrarily shaped thermal cloaks can be designed using homogeneous anisotropic materials only. We construct two polyhedral cloaks and show numerically that they successfully thermally conceal objects after a certain lapse of time. We then demonstrate that cloaks with curved boundaries can also be obtained using our approach. It is further shown how geometrical parameters affect the material properties and cloaking performances. One can flexibly tune the cloaking performances based on practical requirements and material availabilities. We analyze effectiveness of a polygonal cloak composed of an alternation of homogeneous isotropic layers, and note that cloaking deteriorates at short times. We finally sketch an approach to realize 3D homogenized thermal metamaterials.

Keywords: Heat cloaking; Linear mapping transformation; Arbitrarily shaped

Received 8 October 2019, Accepted 26 November 2019

DOI: $10.30919 /$ esee $8 \mathrm{c} 361$

\section{Introduction}

Heat cloaking refers to making an object invisible to heat flows and is achieved by surrounding the object with a predefined heat cloak. It is inspired by the initial works on invisibility of electromagnetic waves. ${ }^{1-3}$ Beyond electromagnetism, ${ }^{4-7}$ cloaking is widely applied to elastic waves, ${ }^{8-13}$ acoustics, ${ }^{14-16}$ and diffusion fields. ${ }^{17-20}$ In our paper we focus on heat cloaking. Fan et. al. ${ }^{21}$ exploited shaped graded materials where heat energy was manipulated to transfer from regions of low temperature to regions of high temperature. Guenneau et. al. ${ }^{22}$ first proposed a transformed coordinate approach of thermal cloaking in the heat equation and established multilayered structures with isotropic materials, which was verified later by experiments. ${ }^{23-26}$ Researchers have then made great achievements on heat manipulation with various thermal metadevices. ${ }^{27-36}$ The pioneering works mainly focused on regular shaped cloaks, i.e. cylindrical and spherical cloaks. Cloaks with more complicated boundaries were then studied in the field of electromagnetism and acoustics. ${ }^{37-44}$ These transforms were later introduced for heat diffusion fields. ${ }^{45}$ The obtained heat cloaks were inhomogeneous and anisotropic which is diffcult to realize in practice. Some researchers studied non regular-shaped cloaks and established systems with homogeneous parts, ${ }^{46-48}$ but they only dealt with limited geometries. Han et.al. designed arbitrarily polygonal cloaks with homogeneous and non-singular parameters, ${ }^{49}$ which was realized by Liu et.al. with four kinds of natural bulk materials in bilayer configuration throughout. ${ }^{50} \mathrm{Xu}$ et. al. designed arbitrary polygonal thermal harvesting schemes using the linear mapping function. ${ }^{51-52} \mathrm{Li}$ et.al. extended these works to three dimensions and proposed a multistep transformation approach for a tetrahedral cloak. ${ }^{53}$ However, in Li's nice work, the transformation for a tetrahedral cloak is realized in three steps. The transformation should be conducted in a certain order as the later transformation step is dependent on the former one. We propose instead a one-step linear mapping for

\footnotetext{
${ }^{1}$ National Key Laboratory of Science and Technology on Advanced Composites in Special Environments, Harbin Institute of Technology, Harbin 150001, China

${ }^{2}$ Institut FEMTO-ST, CNRS, Université de Bourgogne Franche-Comté, Besançon 25000, France

${ }^{3}$ Institute of Advanced Structure Technology, Beijing Institute of Technology, Beijing 100081, China

${ }^{4}$ Aix-Marseille Univ, CNRS, Centrale Marseille, Institut Fresnel, Marseille, France

*Email: fanggd@hit.edu.cn
} 
polyhedral cloaks where each transformation is independent. Homogeneous material parameters are obtained in this process, facilitating practical realizations. It is known that an arbitrary shaped cloak can be approached by polyhedra, but a quantitative analysis based on this approximation was rarely considered. ${ }^{50-53}$ We first show how cloaks with curved boundaries (regular spherical cloak and cylindrical cloak as illustrative cases here) are approximated by polyhedra and we demonstrate the good cloaking performances of the approximated cloaks. For non-regular shaped cloaks, the convergence behavior in homogenization process hasn't been studied, and this paper primarily focuses on this problem. We study the convergence behavior of a multi-layered polygonal cloak in both steady-state and transient state cases. Influences of the cloak's shape and lapse of time on the convergence rate are numerically established. Finally, we propose an approach to realize $3 \mathrm{D}$ homogenized thermal metamaterials.

\section{Methods}

We first consider a linear transformation from the tetrahedral domain in virtual space with local coordinate axes $(x, y, z)$ to the physical tetrahedral space with local coordinate axes $\left(x^{\prime}, y^{\prime}, z^{\prime}\right)$ (see Fig. 1) by

$$
\left(\begin{array}{l}
x^{\prime} \\
y^{\prime} \\
z^{\prime}
\end{array}\right)=\left(\begin{array}{lll}
a_{11} & a_{12} & a_{13} \\
a_{21} & a_{22} & a_{23} \\
a_{31} & a_{32} & a_{33}
\end{array}\right)\left(\begin{array}{l}
x \\
y \\
z
\end{array}\right)+\left(\begin{array}{l}
a_{4} \\
a_{5} \\
a_{6}
\end{array}\right),
$$

where the coefficients $a_{i j}$ and $a_{h}$ are constant. Substituting four groups of corresponding vertices in Eq. 1, we get 12 equations for the 12 coefficients. By solving the 12 equations, we find the transformation coefficients as

$$
\left[\begin{array}{lll}
a_{11} & a_{12} & a_{13} a_{4} \\
a_{21} & a_{22} & a_{23} a_{5} \\
a_{31} & a_{32} & a_{33} a_{6}
\end{array}\right]=\left[\begin{array}{ccc}
x_{A}^{\prime} & x_{B}^{\prime} & x_{C}^{\prime} x_{D}^{\prime} \\
y_{A}^{\prime} & y_{B}^{\prime} & y_{C}^{\prime} y_{D}^{\prime} \\
z_{A}^{\prime} & z_{B}^{\prime} & z_{C}^{\prime} z_{D}^{\prime}
\end{array}\right]\left[\begin{array}{cccc}
x_{A}^{\prime} & x_{B}^{\prime} & x_{C}^{\prime} & x_{D}^{\prime} \\
y_{A}^{\prime} & y_{B}^{\prime} & y_{C}^{\prime} & y_{D}^{\prime} \\
z_{A}^{\prime} & z_{B}^{\prime} & z_{C}^{\prime} & z_{D}^{\prime} \\
1 & 1 & 1 & 1
\end{array}\right]^{-1} .
$$

The transient heat conduction equation without sources can be written as

$$
\nabla \cdot(k \nabla T)-\rho c \frac{\partial T}{\partial t}=0,
$$

where $T$ represents the temperature, $k$ and $\rho c$ are thermal conductivity (in unit of $W \cdot m^{-1} \cdot K^{-1}$ ) and the product of density by heat capacity (in unit of $J \cdot \mathrm{Kg}^{-1} \cdot \mathrm{K}^{-1}$ ), respectively. According to Ref. [22], this equation is form-invariant under coordinate transformation. Note that the transformed parameters $\operatorname{are}^{21-22}$

$$
k^{\prime}=\frac{J k J^{T}}{\operatorname{det}(J)} \text { and }(\rho c)^{\prime}=\frac{\rho c}{\operatorname{det}(J)},
$$

where

$$
J=\left(\begin{array}{lll}
a_{11} & a_{12} & a_{13} \\
a_{21} & a_{22} & a_{23} \\
a_{31} & a_{32} & a_{33}
\end{array}\right) .
$$

Now, the coefficient matrix $J$ is a constant matrix which is independent of $x, y$ and $z$. As a result, material properties derived from Eq. 4 are homogeneous.

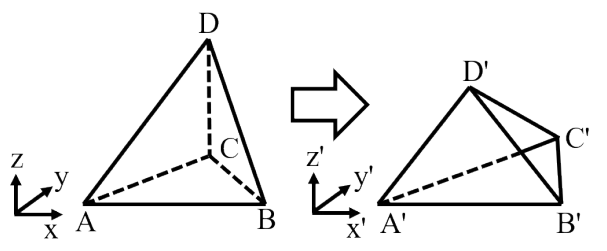

Fig. 1 Coordinate transformation between two tetrahedra. The tetrahedral domain in virtual space (left) is mapped to a physical tetrahedral space (right) by linear transformation.

In Fig. 2 we show a regular tetrahedral cloak presented by Ref. [53], where the shaded domain denotes the designed domain (cloaking domain) while tetrahedron $O A_{1} B_{1} C_{1}$ represents the domain to be cloaked (cloaked domain). For clarity, we select the edge $\mathrm{OA}_{2}$ to be along the $x$-axis, and the face $\mathrm{OA}_{2} B_{2}$ in the xoy plane. We further divide the polyhedral cloaking domain into three tetrahedral segments (marked in different colour in Fig. 2) and apply linear coordinate transformation to each segment. Tetrahedron $A_{1} A_{2} B_{2} C_{2}$, $A_{1} B_{1} B_{2} C_{2}$ and $A_{1} B_{1} C_{1} C_{2}$ in physical space are mapped from $A_{0} A_{2} B_{2} C_{2}, A_{0} B_{0} B_{2} C_{2}$ and $A_{0} B_{0} C_{0} C_{2}$ in virtual space (outlined by green lines in Fig. 2), respectively. Since all the points are located on three edges, we define $O A_{0}=\lambda_{l} O A_{2}, O A_{1}=$ $\lambda_{2} O A_{2}, O B_{0}=\mu_{1} O B_{2}, O B_{1}=\mu_{2} O B_{2}, O C_{0}=\varepsilon_{1} O C_{2} \quad$ and $O C_{1}=\varepsilon_{2} O C_{2}$, where $\lambda_{i}, u_{i}$ and $\varepsilon_{i}$ are all known constants. In the transformation process, the physical space and virtual space are defined by the same coordinate system.

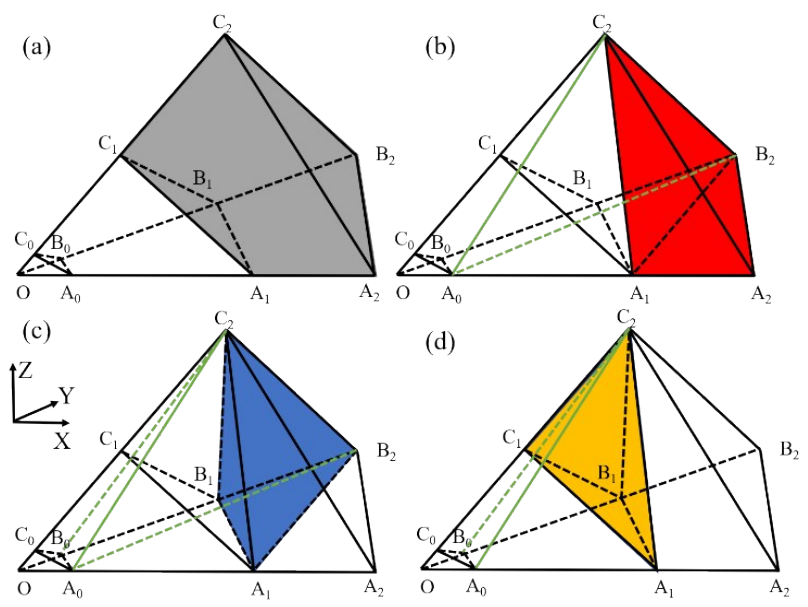

Fig. 2 A general tetrahedron cloak and the sub-domains of the cloaking domain.

In Ref. [53], the transformation for a tetrahedron cloak is completed by multi-steps. The three segments should be mapped in a specific order as the latter transformation step is 
dependent on the former one. In our method, the transformations are all conducted by one step and each transformation is independent. Without losing generality, we first map the points in tetrahedron $A_{0} A_{2} B_{2} C_{2}$ to the points in tetrahedron $A_{1} A_{2} B_{2} C_{2}$. Vertices of the given polyhedron in virtual space $A_{0}\left(\lambda_{1} x_{A_{2}}, 0,0\right), A_{2}\left(x_{A_{2}}, 0,0\right), B_{2}\left(x_{B_{2}}, x_{B_{2}}, 0\right)$ and $C_{2}\left(x_{C_{2}}, y_{C_{2}}, z_{C_{2}}\right)$ are mapped to $A_{1}\left(\lambda_{2} x_{A_{2}}, 0,0\right)$, $A_{2}\left(x_{A_{2}}, 0,0\right), B_{2}\left(x_{B_{2}}, y_{B_{2}}, 0\right)$ and $C_{2}\left(x_{C_{2}}, y_{C_{2}}, z_{C_{2}}\right)$ in physical space, respectively. Substituting these four typical coordinates in Eq. 1, we get the transformation from tetrahedron $A_{0} A_{2} B_{2} C_{2}$ to tetrahedron $A_{1} A_{2} B_{2} C_{2}$ by

$$
\left(\begin{array}{l}
x_{1}^{\prime} \\
y_{1}^{\prime} \\
z_{1}^{\prime}
\end{array}\right)=\left(\begin{array}{lll}
b_{11} & b_{12} & b_{13} \\
b_{21} & b_{22} & b_{23} \\
b_{31} & b_{32} & b_{33}
\end{array}\right)\left(\begin{array}{l}
x_{1} \\
y_{1} \\
z_{1}
\end{array}\right)+\left(\begin{array}{l}
b_{4} \\
b_{5} \\
b_{6}
\end{array}\right),
$$

where

$$
\begin{gathered}
b_{11}=\frac{1-\lambda_{2}}{1-\lambda_{1}}, \\
b_{12}=\frac{\lambda_{2}-\lambda_{1}}{1-\lambda_{1}} \frac{y_{B_{2}}-x_{B_{2}}}{y_{B_{2}}}, \\
\mathrm{~b}_{13}=-\frac{\lambda_{2}-\lambda_{1}}{1-\lambda_{1}} \frac{x_{B_{2}} y_{C_{2}}-x_{C 2} y}{B_{2}+x_{A_{2}}\left(y_{B_{2}}-y_{C_{2}}\right)} \\
y_{B_{2}} z_{C_{2}} \\
b_{4}=\frac{\lambda_{2}-\lambda_{1}}{1-\lambda_{1}} x_{A_{2}}, \\
b_{22}=b_{33}=1, \\
b_{21}=b_{23}=b_{31}=b_{32}=b_{5}=b_{6}=0 .
\end{gathered}
$$

Similarly, the points in tetrahedron $A_{0} B_{0} B_{2} C_{2}$ are mapped onto tetrahedron $A_{1} B_{1} B_{2} C_{2}$ by

$$
\left(\begin{array}{l}
x_{2}^{\prime} \\
y_{2}^{\prime} \\
z_{2}^{\prime}
\end{array}\right)=\left(\begin{array}{lll}
c_{11} & c_{12} & c_{13} \\
c_{21} & c_{22} & c_{23} \\
c_{31} & c_{32} & c_{33}
\end{array}\right)\left(\begin{array}{l}
x_{2} \\
y_{2} \\
z_{2}
\end{array}\right)+\left(\begin{array}{l}
c_{4} \\
c_{5} \\
c_{6}
\end{array}\right) \text {, }
$$

where

$$
\begin{gathered}
c_{11}=\frac{\lambda_{2}}{\lambda_{1}}-\frac{x_{B_{2}}}{\lambda_{1} x_{A_{2}}} \frac{u_{2}-u_{1}}{1-u_{1}}, \\
c_{12}=\frac{x_{B_{2}}}{y_{B_{2}}}\left[\frac{u_{2}-u_{1}}{1-u_{1}}\left(\frac{x_{B_{2}}}{\lambda_{1} x_{A_{2}}}-1\right)-\frac{\lambda_{2}-\lambda_{1}}{\lambda_{1}}\right], \\
c_{13}=\frac{u_{2}-u_{1}}{1-u_{1}}\left[\frac{x_{B_{2}}}{z_{C_{2}}}\left(\frac{x_{C_{2}}}{\lambda_{1} x_{A_{2}}}-1\right)-\frac{x_{B_{2}} y_{C_{2}}}{y_{B_{2}} z_{C_{2}}}\left(\frac{x_{B_{2}}}{\lambda_{1} x_{A_{2}}}-1\right)\right]- \\
\frac{\lambda_{2}-\lambda_{1}}{\lambda_{1}} \frac{x_{C_{2}} y_{B_{2}}-x_{B_{2}} y_{C_{2}}}{y_{B_{2}} z_{C_{2}}}, \\
c_{4}=\frac{u_{2}-u_{1}}{1-u_{1}} x_{B_{2}}, \\
c_{21}=-\frac{u_{2}-u_{1}}{1-u_{1}} \frac{y_{B_{2}}}{\lambda_{1} x_{A_{2}}},
\end{gathered}
$$

$$
\begin{gathered}
c_{22}=\frac{u_{2}-u_{1}}{1-u_{1}}\left(\frac{x_{B_{2}}}{\lambda_{1} x_{A_{2}}}-1\right)+1, \\
c_{23}=\frac{u_{2}-u_{1}}{1-u_{1}}\left[\frac{y_{B_{2}}}{z_{C_{2}}}\left(\frac{x_{C_{2}}}{\lambda_{1} x_{A_{2}}}-1\right)-\frac{y_{C_{2}}}{z_{C_{2}}}\left(\frac{x_{B_{2}}}{\lambda_{1} x_{A_{2}}}-1\right)\right], \\
c_{5}=\frac{u_{2}-u_{1}}{1-u_{1}} y_{B_{2}}, \\
c_{31}=c_{32}=c_{6}=0, \\
c_{33}=1
\end{gathered}
$$

The mapping from points in tetrahedron $A_{0} B_{0} C_{0} C_{2}$ to points in tetrahedron $A_{1} B_{1} C_{1} C_{2}$ is given by

$$
\left(\begin{array}{l}
x_{3}^{\prime} \\
y_{3}^{\prime} \\
z_{3}^{\prime}
\end{array}\right)=\left(\begin{array}{lll}
d_{11} & d_{12} & d_{13} \\
d_{21} & d_{22} & d_{23} \\
d_{31} & d_{32} & d_{33}
\end{array}\right)\left(\begin{array}{l}
x_{3} \\
y_{3} \\
z_{3}
\end{array}\right)+\left(\begin{array}{l}
d_{4} \\
d_{5} \\
d_{6}
\end{array}\right),
$$

where

$$
\begin{gathered}
d_{11}=\frac{\lambda_{2}}{\lambda_{1}}-\frac{\varepsilon_{2}-\varepsilon_{1}}{1-\varepsilon_{1}} \frac{x_{C_{2}}}{\lambda_{1} x_{A_{2}}}, \\
\mathrm{~d}_{12}=\frac{1}{u_{1} y_{B_{2}}}\left[\frac{\varepsilon_{2}-\varepsilon_{1}}{1-\varepsilon_{1}} x_{C_{2}}\left(\frac{u_{1} x_{B_{2}}}{\lambda_{1} x_{A_{2}}}-1\right)+\frac{\lambda_{2} u_{1}-\lambda_{1} u_{2}}{\lambda_{1}} x_{B_{2}}\right], \\
\mathrm{d}_{13}=\frac{\varepsilon_{2}-\varepsilon_{1}}{1-\varepsilon_{1}} \frac{\mathrm{x}_{\mathrm{C}_{2}}}{\mathrm{z}_{\mathrm{C}_{2}}}\left[\frac{\mathrm{x}_{\mathrm{C}_{2}}}{\lambda_{1} \mathrm{x}_{\mathrm{A}_{2}}}-\frac{\mathrm{y}_{\mathrm{C}_{2}}}{\mathrm{u}_{1} \mathrm{y}_{\mathrm{B}_{2}}}\left(\frac{\mathrm{u}_{1} \mathrm{x}_{\mathrm{B}_{2}}}{\lambda_{1} \mathrm{x}_{\mathrm{A}_{2}}}-1\right)-1\right]+ \\
\mathrm{d}_{21}=-\frac{1}{z_{C_{2}}}\left[\frac{\varepsilon_{2}-\varepsilon_{1}}{1-\varepsilon_{1}} \frac{y_{C_{2}}}{\lambda_{1} x_{A_{2}}}, d_{22}=\frac{\lambda_{1} u_{2}}{\lambda_{1}-\varepsilon_{B_{2} y_{C_{2}}}}-\frac{\lambda_{2}-\lambda_{1}}{u_{1} y_{B_{2}}} \frac{y_{C_{2}}}{\lambda_{1}} x_{C_{2}}\right], \\
d_{23}=\frac{\varepsilon_{2}-\varepsilon_{1} x_{B_{2}}}{1-\varepsilon_{1}} \frac{y_{C_{2}}}{z_{C_{2}}}\left[\frac{x_{C_{2}}}{\lambda_{1} x_{A_{2}}}-\frac{y_{C_{2}}}{u_{1} y_{B_{2}}}\left(\frac{u_{1} x_{B_{2}}}{\lambda_{1} x_{A_{2}}}-1\right)-1\right), \\
\mathrm{d}_{31}=-\frac{\varepsilon_{2}-\varepsilon_{1}}{1-\varepsilon_{1}} \frac{z_{C_{2}}}{\lambda_{1} x_{A_{2}}}, d_{32}=\frac{\varepsilon_{2}-\varepsilon_{1}}{1-\varepsilon_{1}} \frac{z_{C_{2}}}{u_{1} y_{B_{2}}}\left(\frac{u_{1} x_{B_{2}}}{\lambda_{1} x_{A_{2}}}-1\right), \\
d_{33}=\frac{\varepsilon_{2}-\varepsilon_{1}}{1-\varepsilon_{1}}\left[\frac{x_{C_{2}}}{\lambda_{1} x_{A_{2}}}-\frac{y_{C_{2}}}{u_{1} y_{B_{2}}}\left(\frac{u_{1} x_{B_{2}}}{\lambda_{1} x_{A_{2}}}-1\right)-1\right]+1, \\
d_{4}=\frac{\varepsilon_{2}-\varepsilon_{1}}{1-\varepsilon_{1}} x_{C_{2}}, \\
d_{5}=\frac{\varepsilon_{2}-\varepsilon_{1}}{1-\varepsilon_{1}} y_{C_{2}}, \\
d_{6}=\frac{\varepsilon_{2}-\varepsilon_{1}}{1-\varepsilon_{1}} z_{C_{2}}
\end{gathered}
$$

Substituting the obtained transformation matrices $J_{1}, J_{2}$ and $J_{3}$ into Eq. (4), we get the transformed material properties for each segment. In the above transformation, we assume the tetrahedron $O A_{0} B_{0} C_{0}$ is a void and we map it to the void tetrahedron $O A_{1} B_{1} C_{1}$, thus the object inside $O A_{1} B_{1} C_{1}$ is cloaked. Remark that we can choose to fill the cloaked domain with a medium. Following Ref. [54], the transformed 
parameters for the cloaked domain are given by

$k_{\text {in }}^{\prime}=\frac{\lambda_{1} u_{1} \varepsilon_{1}}{\lambda_{2} u_{2} \varepsilon_{2}} \operatorname{diag}\left(\left(\frac{\lambda_{2}}{\lambda_{1}}\right)^{2},\left(\frac{u_{2}}{u_{1}}\right)^{2},\left(\frac{\varepsilon_{2}}{\varepsilon_{1}}\right)^{2}\right),(\rho c)_{\text {in }}^{\prime}=\frac{\lambda_{1} u_{1} \varepsilon_{1}}{\lambda_{2} u_{2} \varepsilon_{2}}(\rho c)$.

\section{Results and discussion}

\subsection{An Octahedral Cloak}

We first build an octahedral cloak where $O A_{2}, O B_{2}$ and $O C_{2}$ lie on the $x, y$ and $z$ axes, respectively (see Fig. (3a)). The domain to be cloaked is depicted in blue while the cloaking domain is depicted in the grey. We split the top and bottom halves into four symmetric tetrahedra. Each tetrahedron is divided into three segments and then mapped following the method outlined in above section. In the octahedral cloak, we set $\lambda_{1}=\mu_{1}=$ $\varepsilon_{1}=0.01$ and $\lambda_{2}=\mu_{2}=\varepsilon_{2}=0.5$. For convenience, we normalize the material properties with respect to unit conductivity and heat capacity in background medium. The octahedral cloak is totally divided into 8 tetrahedra and hence 24 segments. Owing to symmetry, we only present material properties of the three unique segments, which are

$$
\left\{\begin{array}{c}
k_{1}^{\rho}=\operatorname{diag}(0.38,1.98,2.58),(\rho c)_{1}^{\prime}=1.98 \\
k_{2}^{\rho}=\operatorname{diag}(39.6,6.5,10.8),(\rho c)_{2}^{\prime}=0.04 \\
k_{3}^{\rho}=\operatorname{diag}(1.98,5.86,0.0001),(\rho c)_{3}^{\prime}=0.0008
\end{array}\right.
$$

Properties of other parts can be easily obtained using symmetries. Numerical simulations are then conducted with the finite element package COMSOL Multiphysics. We set temperatures on the left boundary (the boundary in negative $x$ axis) and right boundary (the boundary in positive $x$ axis) as $T_{L}=2 \mathrm{~K}$ and $T_{R}=1 \mathrm{~K}$, respectively. Other boundaries are thermally insulated to ensure that conduction is the dominant heat transfer mechanism in this case. For clarity, we only show the cloaking performances on two typical sections where the white lines are iso-thermal lines. Simulation results for the steady-state case (see Fig. (3b)) show that heat fluxes pass through the object and return to their original trajectory. As a result, the external heat field is not disturbed. For an outside observer, it seems that the heat is transferred through a homogeneous medium.
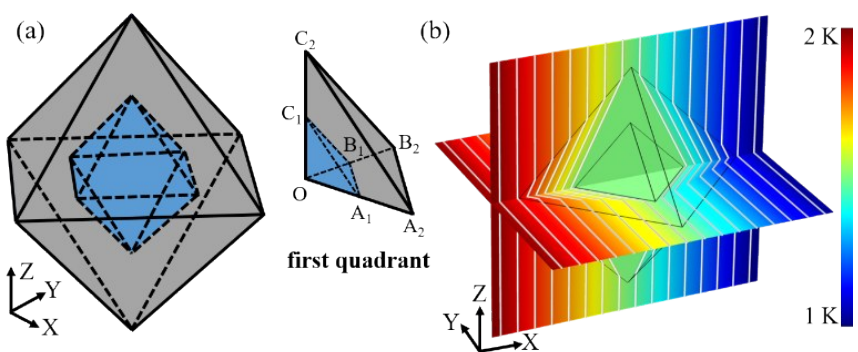

Fig. 3 Schematic of (a) an octahedral heat cloak and (b) its steady-state cloaking performance.
In the dynamic case, we add perfectly matched layer on the right boundary and apply different time-dependent thermal sources on the left boundary, which are $T_{1}=T_{0} \exp \left(-t^{2} / 2\right)+$ 1 and $T_{2}=T_{0} \sin (\pi t / 2)+2$. We set $T_{0}=1 K$ and conduct simulations. Good cloaking performances are observed for both cases at different times. In Fig. 4, we set continuously decreasing temperatures on the left boundary and observe good cloaking effects all the time. In Fig. 5, we apply periodic thermal boundary on the left and notice that heat energy transfers from left to right. During the transfer process, good cloaking performance is observed at changing thermal profiles.

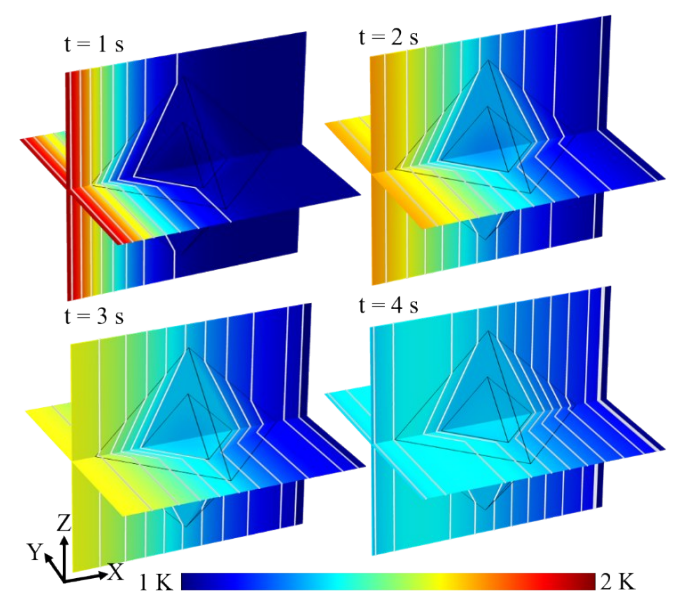

Fig. 4 Dynamic cloaking performances of the octahedral cloak with the left boundary $T_{1}=T_{0} \exp \left(-t^{2} / 2\right)+1$.

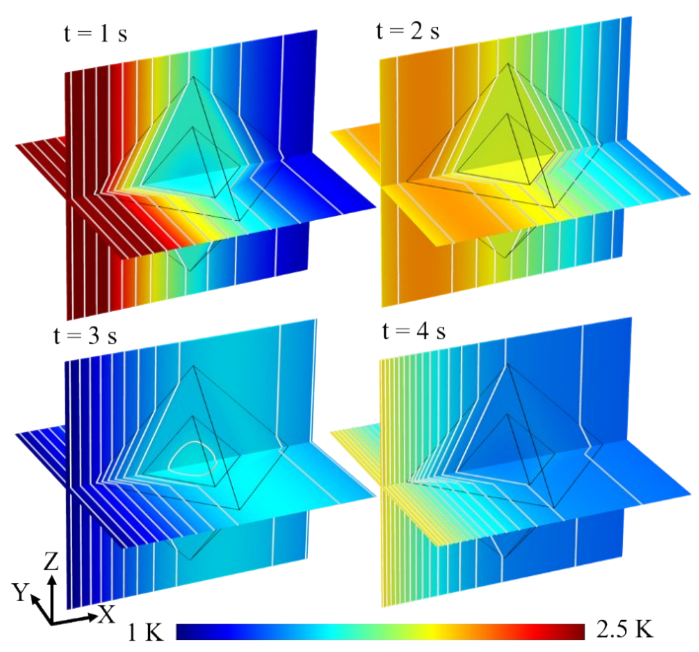

Fig. 5 Dynamic cloaking performances of the octahedral cloak with the left boundary $T_{2}=T_{0} \sin (\pi t / 2)+2$.

\subsection{A Polyhedral Cloak with 32 Faces}

We also designed more complicated cloaks with 32 faces (see Fig. 6). Similar to the octahedral cloak, the polyhedral cloak is totally divided into 32 tetrahedra and hence 96 segments. We set $\lambda_{1}=\mu_{1}=\varepsilon_{1}=0.01$ and $\lambda_{2}=\mu_{2}=\varepsilon_{2}=0.5$. Owing to 
symmetry, we only consider the parts in the first quadrant as the illustrative case and present material properties of the unique segments. In the first quadrant, the cloak is divided in four parts and each part is further divided into 12 segments, of which two parts (6 segments) are unique. For the central part $O \mathrm{~B}_{2} \mathrm{C}_{2} E_{2}$, the properties of the three segments are

$$
\left\{\begin{array}{c}
k_{1}^{\rho}=\operatorname{diag}(0.51,1.98,1.98),(\rho c)_{1}^{\prime}=1.98 \\
k_{2}^{\rho}=\operatorname{diag}(291.14,0.05,0.03),(\rho c)_{2}^{\prime}=0.04 . \\
k_{3}^{\rho}=\operatorname{diag}(291.14,0.05,0.03),(\rho c)_{3}^{\prime}=0.0008
\end{array} .\right.
$$

The other three parts on the edges have similar forms. Therefore, we present the typical part $\mathrm{OA}_{2} B_{2} C_{2}$ with one edge on the $x$-axis:

$$
\left\{\begin{array}{l}
k_{1}^{\rho}=\operatorname{diag}(2.286,0.437,1.977),(\rho c)_{1}^{\prime}=1.98 \\
k_{2}^{\rho}=\operatorname{diag}(146.755,0.007,0.035),(\rho c)_{2}^{\prime}=0.04 \\
k_{3}^{\rho}=\operatorname{diag}(2.647,0.002,1.98),(\rho c)_{3}^{\prime}=0.0008
\end{array}\right.
$$

Simulation results for the steady-state case (see Fig. 6b) again show that this polyhedral cloak achieves good cloaking. In the dynamic case, we apply same boundary conditions as those of the octahedral cloak. In Fig. 7 and Fig. 8, good cloaking performances are observed at changing thermal profiles.

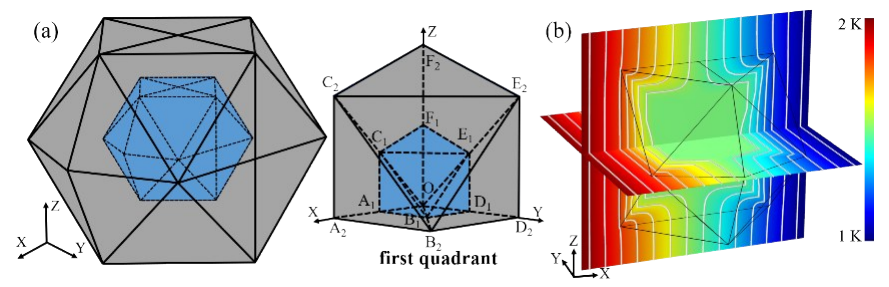

Fig. 6 Schematic of (a) a polyhedral heat cloak and (b) its steady-state cloaking performance.



Fig. 7 Dynamic cloaking performances of the polyhedral cloak with the left boundary $T_{1}=T_{0} \exp \left(-t^{2} / 2\right)+1$.



Fig. 8 Dynamic cloaking performances of the polyhedral cloak with the left boundary $T_{2}=T_{0} \sin (\pi t / 2)+2$.

\subsection{Cloaks with Curved Boundaries}

We have shown in the above section that the proposed method can be applied to polyhedral cloaks with more faces. Theoretically, an arbitrarily shaped cloak with curved boundaries can be approximated by an arbitrarily large number of polyhedral cloaks, hence our approach applies to a wide range of geometries. For cloaks with curved boundaries, they are firstly approximated as series of tetrahedra and then divided into homogeneous segments. In this process, we approximate the curves by piecewise lines and apply a linear transformation on each segment. As an illustration, we show in this section how spherical cloaks and cylindrical cloaks can be obtained by our method. We build an arbitrary tetrahedron, three vertices of which lie on the surface of a sphere and one vertex lies on the centre (see Fig. 9). We make simplifying assumptions that $\alpha=\beta=\gamma=\theta, \lambda_{1}=\mu_{1}=\varepsilon_{1}=\eta_{1}, \lambda_{2}=\mu_{2}=\varepsilon_{2}=\eta_{2}$.

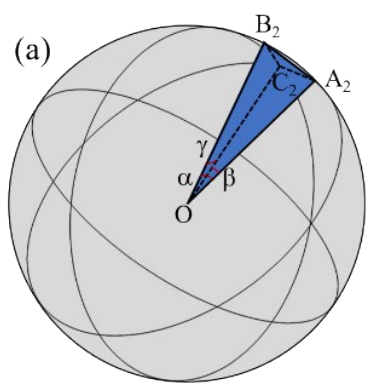

(b)

Fig. 9 Diagram demonstration of (a) a tetrahedron derived from the sphere and (b) the studied tetrahedron with simplifying conditions.

The tetrahedron will increasingly approximate parts of the sphere as $\theta$ decreases. It is also worth noting that the second and third parts (denoted as $A_{0} B_{0} B_{2} C_{2}$ and $A_{0} B_{0} C_{0} C_{2}$ in Fig. 9, respectively) occupy increasingly small relative fractions, and 
can be negligible as $\theta$ approaches 0 . Therefore, we mainly focus on the first part and easily get the material properties as

$$
\mathrm{k}^{\prime}=\left(\begin{array}{cc}
\frac{1-\lambda_{2}}{1-\lambda_{1}}+\frac{2\left(1-\eta_{1}\right)}{1-\eta_{2}}\left(\frac{\eta_{2}-\eta_{1}}{1-\eta_{1}}\right)^{2} \tan (\theta) & -\frac{\eta_{2}-\eta_{1}}{1-\eta_{2}} \tan (\theta) \\
-\frac{\eta_{2}-\eta_{1}}{1-\eta_{2}} \tan (\theta) & \frac{1-\eta_{1}}{1-\eta_{2}} \\
-\frac{\eta_{2}-\eta_{1}}{1-\eta_{2}} \tan (\theta) & 0
\end{array}\right.
$$

As $\theta$ approaches 0 , the heat conductivity in the principal direction can be expressed as

$$
k_{s}^{\prime}(\theta \rightarrow 0)=\operatorname{diag}\left(\frac{1-\eta_{2}}{1-\eta_{1}}, \frac{1-\eta_{1}}{1-\eta_{2}}, \frac{1-\eta_{1}}{1-\eta_{2}}\right) .
$$

We build spherical cloaks and conduct simulations when $\eta_{2}=0.5$ and $\eta_{1}$ is varied. The results in Fig. 10 show that we can still achieve good cloaking effects using the constitutive properties derived from the polyhedral cloaks. It is also observed that the cloaking performance deteriorates with increasing $\eta_{1}$, as more and more heat fluxes invade the cloaked domain. When $\eta_{1}=0.5$, there is no physical transformation and hence no cloaking effects at all.

Similarly, we derive the material properties of a cylindrical cloak from the polyhedral approach as

$$
k_{c}^{\prime}(\theta \rightarrow 0)=\operatorname{diag}\left(\frac{1-\eta_{2}}{1-\eta_{1}}, \frac{1-\eta_{1}}{1-\eta_{2}}, \frac{1-\eta_{1}}{1-\eta_{2}}\right) .
$$

We build cylindrical cloaks and conduct simulations where $\eta_{1}=0.5$ and $\eta_{1}$ is varied. Again, we show in Fig. 11 that the polygonal approach is also applicable to cylindrical cloaks. The parameter $\eta_{1}$ shows similar influence on the cloaking performance. Though smaller $\eta_{1}$ is desirable in order to improve cloaking, one should consider other influential factors in realizations, e.g. material anisotropy and availability. A

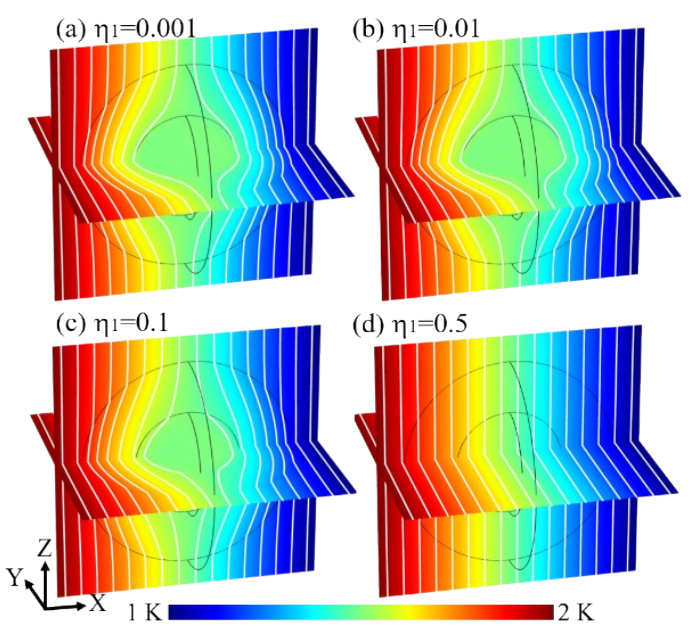

Fig. 10 Performances of the approximated spherical cloak for (a) $\eta_{1}=0.001$, (b) $\eta_{1}=$ 0.01 , (c) $\eta_{1}=0.1$ and (d) $\eta_{1}=0.5$. smaller value of $\eta_{1}$ means more difficulties during realization, which we will discuss in the next section.

$$
\left.\begin{array}{c}
-\frac{\eta_{2}-\eta_{1}}{1-\eta_{2}} \tan (\theta) \\
0 \\
\frac{1-\eta_{1}}{1-\eta_{2}}
\end{array}\right),(\rho c)^{\prime}=\frac{1-\eta_{1}}{1-\eta_{2}}
$$

\subsection{Influence of Geometrical Parameters}

In the proposed method, we map a small hollow tetrahedron $O A_{0} B_{0} C_{0}$ to tetrahedron $O A_{1} B_{1} C_{1}$, thus the object inside the void tetrahedron is cloaked. As we consider the transformation from a hollow tetrahedron instead of a point, we obtain nonsingular material properties. This is inspired by the regularized transform first proposed in Ref. [54]. We choose the octahedral cloak in above section to illustrate the effect of geometry parameters on cloaking.

Without lack of generality, we set $\lambda_{2}=\mu_{2}=\varepsilon_{2}=0.5$ and $\lambda_{1}=\mu_{1}=\varepsilon_{1}=\eta_{1}$ where $\eta_{1}$ is a variable. A perturbation index is introduced to characterize the cloaking performance

$$
M_{V}(t)=\frac{\int_{\Omega}\left|T(t, x, y, z)-T_{r}(t, x, y, z)\right| d \Omega}{\int_{\Omega} d \Omega},
$$

where $\Omega$ denotes the probe domain of external thermal fields and $T_{r}$ represents temperatures of the homogeneous medium. The index $M_{V}$ reveals all perturbations of the external heat profile. A lower value of $M_{V}$ denotes better cloaking performance. We use $M_{V}^{1}$ and $M_{V}^{2}$ to reveal the case of only a small hollow tetrahedron and a proposed cloak, respectively. We can observe in Fig. 12(a) that the two values are always identical, demonstrating the effectiveness of our design. Besides, it can be seen that increasing $\eta_{1}$ results in larger

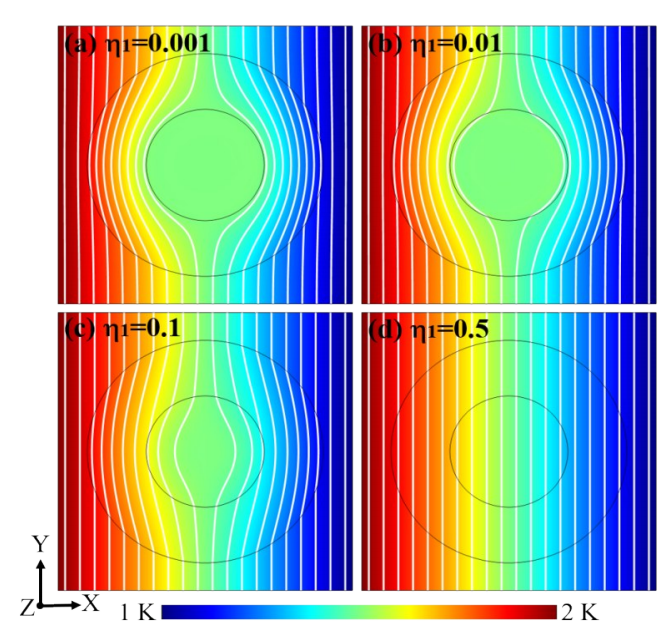

Fig. 11 Performances of the approximated cylindrical cloak for (a) $\eta_{1}=0.001$, (b) $\eta_{1}=$ 0.01 , (c) $\eta_{1}=0.1$ and (d) $\eta_{1}=0.5$. 
perturbations and thus worsens the effects of cloaking. When $\eta_{1}$ equals the limiting value of 0.5 , namely when the tetrahedron $O A_{0} B_{0} C_{0}$ coincides with tetrahedron $O A_{1} B_{1} C_{1}$, there is no cloaking effect at all.

It seems that we should make $\eta_{1}$ as small as possible to achieve better cloaking performance. However, the transformed material properties are always beyond the range of natural materials. Geometry parameters determine the material properties and they should be tailored to make the cloaks easier to realize. As we can see in Fig. 12, a smaller $\eta_{1}$ means a higher degree of material anisotropy, which poses more difficulties in practical realization. Some compromise between performance and anisotropy is needed, and one quantity is necessarily improved at the cost of trading off the other one.
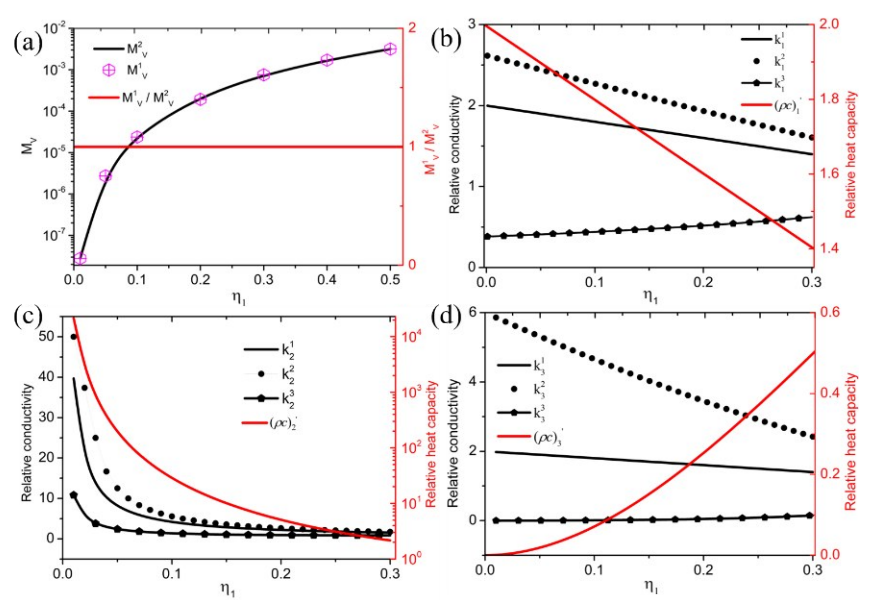

Fig. 12 Effects of the geometry parameters on cloaking performances and material properties.

\subsection{Towards Effectiveness of Homogenized Multi-Layered Cloaks}

We have obtained polyhedral heat cloaks with non-singular homogeneous but anisotropic material properties. In real applications, the material parameters still need to be fulfilled usually by layered structures. For this purpose, we start from alternations of isotropic thin layers with respective constitutive properties $\left(k_{1}^{\prime}, \rho_{1}^{\prime} c_{1}^{\prime}\right)$ and $\left(k_{2}^{\prime}, \rho_{2}^{\prime} c_{2}^{\prime}\right)$. We study a polygonal cloak and derive material properties by the proposed approach. As the designed polygonal cloak is piecewise homogeneous, the constitutive properties are all locally constant and do not depend on the position. The required heat conductivity and product of density by heat capacity can be approximated by ${ }^{22}$

$$
k_{\perp}^{\prime}=\frac{\left(l_{1}+l_{2}\right) k_{1}^{\prime} k_{2}^{\prime}}{l_{1} k_{1}^{\prime}+l_{2} k_{2}^{\prime}}, k_{/ /}^{\prime}=\frac{l_{1} k_{1}^{\prime}+l_{2} k_{2}^{\prime}}{l_{1}+l_{2}}, \rho^{\prime} c^{\prime}=\frac{l_{1} \rho_{1}^{\prime}+l_{2} \rho_{2}^{\prime}}{l_{1}+l_{2}},
$$

where $l_{1}$ and $l_{2}$ are thickness ratios of the two layers. For the cloak in Fig. 13, geometrical parameters are $\left|O A_{2}\right|=\left|O B_{2}\right|=$ $1,\left|O A_{1}\right|=\left|O B_{1}\right|=0.5$ and normalized material properties are $\mathrm{k}_{1}=4.96, \mathrm{k}_{2}=0.20, \mathrm{k}_{3}=392.03, k_{4}=0.0026$. Perpendicular vectors of the layers are $V_{\perp}^{1}=(-0.85,-0.52)^{T}$ and $\mathrm{V}_{\perp}^{2}=$ $(-0.70,-0.71)^{T}$, respectively.

As Petiteau et al. [55] proposed, we can determine quantitatively the effectiveness of a cloak by evaluating the standard deviation of the isotherms in the vicinity of the cloak. A theoretically perfect cloak gives a standard deviation value of zero for all isotherms outside the cloak as if the medium were completely homogeneous. In the ideal case, all isotherms outside the cloak are straight. In Fig. 13 we chose the isotherm that passes through vertex $A_{2}$ in the diffusion direction as it is the most difficult to straighten. We performed numerical simulations to check the homogenization convergence as the number of layers $N$ increases. In Fig. 14, increasing effectiveness (decreasing standard deviation) is observed for multi-layered cloaks as the number of thin layers $N$ increases. We obtain the polynomial fit relationship for the convergence rate as

$$
y=-2.2218-1.8269 x+0.5347 x^{2},
$$

where $\mathrm{y}$ is the standard deviation and $x$ is the logarithm of the number of layers: $x=\log (N)$. Eq. 16 has a different form with Petiteau's cylindrical cloak, ${ }^{55}$ demonstrating that the cloak's shape directly affects convergence. Besides, the layered polygonal cloak consists of homogeneous isotropic layers, which is not the case in Petiteau's work where heterogeneous layers are used. This can also have an impact on convergence.

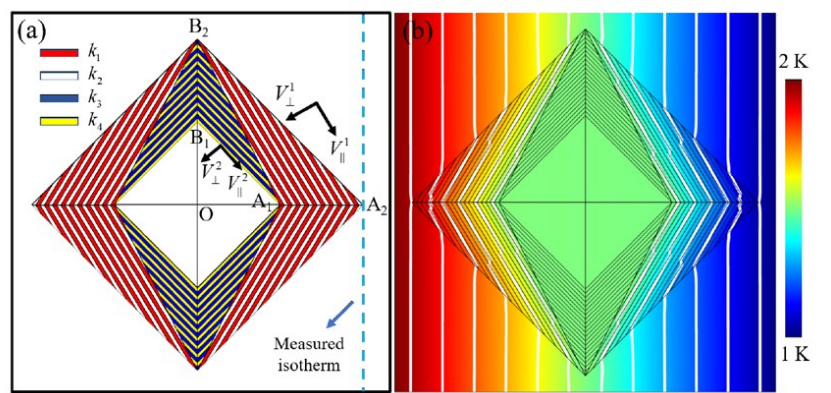

Fig. 13 Two-dimensional polygonal cloak composed of four isotropic materials (left) and the cloaking performances (right).

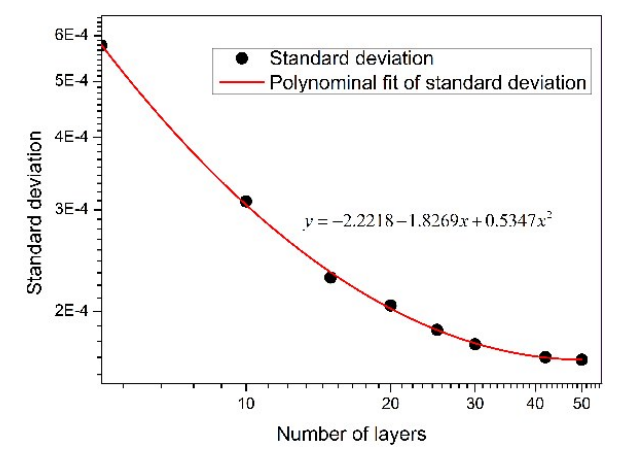

Fig. 14 Logarithm of the standard deviation for the measured isotherm as a function of $\log (N)$. For clarity, the values of $\log (N)$ are replaced by values of $N$. 
Now the goal is to ascertain that the homogenized multilayered cloak mimics the perfect cloak (as built by transformed parameters). We derive the standard deviation of the perfect cloak for the measured isotherm by numerical simulations, and substitute the obtained value $\left(8.61 \times 10^{-16}\right.$ in this case $)$ in Eq. 16. Direct calculation gives the number of layers required to achieve our goal: $N=2.3354 \times 10^{4}$. It is difficult or even impossible to build a nearly perfect cloak with so many layers. However, the convergence analysis provides us with guidance on the number of layers needed for a certain effectiveness tolerance.

We note that in Ref. [56], the following result is established for cloaks with a sufficiently regular (Lipschitz) boundary within a bounded domain $\Omega$ of boundary $\partial \Omega$

Proposition 1: Given $\eta_{1}>0$, there exists a threshold time $t^{*}\left(\eta_{1}\right)<\infty$ such that for all $t \geqslant t^{*}\left(\eta_{1}\right)$

$$
\sqrt{\int_{\Omega \backslash \text { cloak }}\left|T(t, x, y, z)-T_{r}(t, x, y, z)\right|^{2} d \partial \Omega} \leqslant C_{1} \eta_{1}^{D},
$$

where $C_{1}$ is a constant independent of $\eta_{1}$ and $D=2,3$ is dimension of the physical space. This means that after the lapse of a certain threshold time $t^{*}$, the temperature field $T$ outside the cloak should be close to the temperature field $T_{r}$ of the uniformly conducting medium irrespective of the conductivity enclosed in the cloaked region. In other words, boundary measurements on $\partial \Omega$ cannot tell apart $T$ and $T_{r}$.

We first note that in Ref. [57], the following homogenization result is established which can be applied to layered cloaks with a sufficiently regular (Lipschitz) boundary Proposition 2: Given a large enough integer $N>0$, there exists a threshold time $t^{*}(N)<\infty$ such that for all $t \geqslant t^{*}(N)$

$$
\sqrt{\int_{\text {clook }}\left|T(t, x, y, z)-T_{N}(t, x, y, z)\right|^{2} d \Omega} \leqslant C_{2} N^{-1},
$$

where $C_{2}$ is a constant independent of $N, T$ is the temperature field in the ideal cloak and $T_{N}$ that in the layered cloak with $N$ layers.

A direct application of the triangular inequality to Propositions 1 and 2 leads to the following result for a bounded domain $\Omega$ encompassing either a polygonal $(D=2)$ or a polyhedral cloak approximated by isotropic homogeneous layers:

Corollary: Given a small parameter $\eta_{1}$ and a large enough integer $N>0$, there exists a threshold time $t^{*}\left(\eta_{1}, N\right)<\infty$ such that for all $t \geqslant t^{*}\left(\eta_{1}, N\right)$ we have

$$
\sqrt{\int_{\Omega \backslash \text { clook }}\left|T_{r}(t, x, y, z)-T_{N}(t, x, y, z)\right|^{2} d \partial \Omega} \leqslant\left(C_{1} \eta_{1}^{D}+C_{2} N^{-1}\right),
$$

where $T_{r}$ is the temperature field in the homogeneous medium and $T_{N}$ that in the homogeneous medium surrounding the layered cloak with $N$ layers. Using this theoretical result as a guidance, we then compare the effectiveness of the layered cloak and perfect cloak in the transient regime. It is observed in Fig. 15(a) that the standard deviation for a layered cloak first increases and then gradually decreases with the lapse of time, which demonstrates that a layered cloak approximates well an ideal cloak only after a certain lapse of time, whereas at initial times the approximation will be fairly poor. In Fig. 15(b) it is shown that the convergence rate is different at different time steps, demonstrating that in the transient regime the convergence depends on both the lapse of time and the shape of cloaks.


Fig. 15 Standard deviation of the measured isotherm at different time steps with (a) varied time (b) varied number of layers.

We should also note that we use four isotropic materials in the layered polygonal cloak. In the approximation of cloaks with curved shapes, we divide the boundary curves into piecewise line sides and we need four materials for each side. Therefore, the number of heat conductivities needed in the homogenization directly depends on the number of divided sides of a cloak. In the two-dimensional case, we will need $4 n$ different conductivities and thus $4 n$ different types of layers for $n$ sides. As an inhomogeneous spherical or cylindrical cloak can be approximated by a polyhedral cloak with a very large number of sides, this demonstrates that it is always easier to build a polyhedral cloak than a spherical cloak or a cylindrical cloak.

\subsection{Homogenization of 3D Thermal Metamaterials}

Let us finally consider a periodic medium with cubic elementary cells $\eta Y=[0, \eta]^{3}$ of side-length $\eta=1 / N$ where $N$ is a large integer, so that $\eta \ll 1$. Then the solution $T_{\eta}$ of the heat equation with fast oscillating parameters $A_{\eta}=$ $k(x / \eta, y / \eta, z / \eta)$ and $B_{\eta}=\rho(x / \eta, y / \eta, z / \eta) c(x / \eta, y / \eta, z / \eta)$

$$
\nabla \cdot\left(A_{\eta} \nabla T_{\eta}\right)=B_{\eta} \frac{\partial}{\partial t} T_{\eta}
$$

tends, when $\eta$ tends to zero, to the solution $T_{\text {hom }}$ of the following homogenized heat equation ${ }^{58}$

$$
\nabla \cdot\left(\left[A_{\text {hom }}\right] \nabla T_{\text {hom }}\right)=B_{\text {hom }} \frac{\partial}{\partial t} T_{\text {hom }},
$$

where $B_{\text {hom }}=\langle\rho c\rangle_{Y}=\int_{0}^{1} \int_{0}^{1} \int_{0}^{1} \rho(x, y, z) c(x, y, z) d x d y d z$ denotes the average of $\mathrm{B}$ over a periodic cell $Y=[0,1]^{3}$, and $\left[A_{\text {hom }}\right]$ is a $3 \times 3$ matrix given by ${ }^{58}$ 


$$
A_{\text {hom }}=\left(\begin{array}{ccc}
\langle k\rangle_{Y}-\left\langle k \partial_{x} V_{1}\right\rangle_{Y} & -\left\langle k \partial_{x} V_{2}\right\rangle_{Y} & -\left\langle k \partial_{x} V_{3}\right\rangle_{Y} \\
-\left\langle k \partial_{y} V_{1}\right\rangle_{Y} & \langle k\rangle_{Y}-\left\langle k \partial_{y} V_{2}\right\rangle_{Y} & -\left\langle k \partial_{y} V_{2}\right\rangle_{Y} \\
-\left\langle k \partial_{z} V_{1}\right\rangle_{Y} & -\left\langle k \partial_{z} V_{2}\right\rangle_{Y} & \langle k\rangle_{Y}-\left\langle k \partial_{z} V_{3}\right\rangle_{Y}
\end{array}\right) \text {, }
$$

where $\quad \partial x:=\partial / \partial x, \partial y:=\partial / \partial y, \partial z:=\partial / \partial z$ and $V_{1}(x, y, z)$, $V_{2}(x, y, z)$ and $V_{3}(x, y, z)$ are solutions defined up to an additive constant of three auxiliary problems of thermostatic type on the periodic cell $Y:^{.58}$

$$
\left\{\begin{array}{l}
\nabla \cdot\left[k(x, y, z) \nabla\left(V_{1}-x\right)\right]=0 \\
\nabla \cdot\left[k(x, y, z) \nabla\left(V_{2}-y\right)\right]=0 \\
\nabla \cdot\left[k(x, y, z) \nabla\left(V_{3}-z\right)\right]=0
\end{array}\right.
$$

Following this approach, we build a cubic unit cell $Y=$ $[0,1]^{3}$ (medium $A$ ) with cross bars (medium $B$ ) inside as inclusions (see Fig. 16). Medium $A$ is defined as a heat insulating material (air), which means that we have just one medium, and then all the integrals are taken over $Y \backslash A$. Without loss of generality, we only choose the segment $k_{1}^{\rho}=$ diag $(0.38,1.98,2.58)$ of the octahedral cloak in above section as the studied part and design homogenized cells. Normalized heat conductivity of medium $B$ is defined as $k_{B}=400$. We solved the auxiliary problems and calculate the homogenized conductivity, then we got $\left(L_{x}, L_{y}, L_{z}\right)=$ $(0.03,0.07,0.08)$ after parameter sweeping and some optimization. It is noted that our designed elementary cell is not the unique solution to homogenization of $3 \mathrm{D}$ thermal metamaterials, one can achieve the homogenization by other structures and materials using some inverse homogenization techniques. ${ }^{59}$

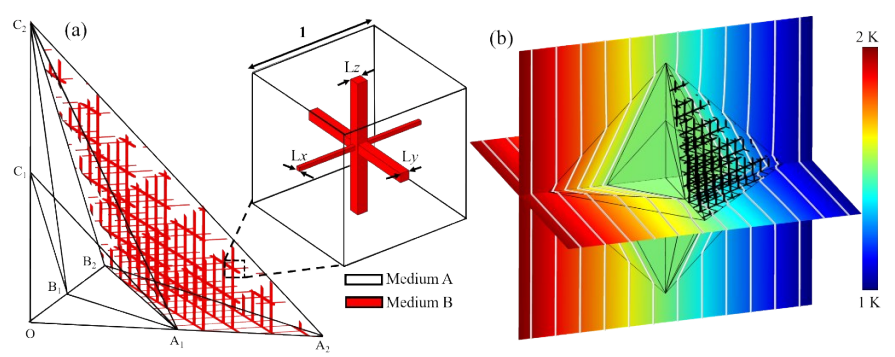

Fig. 16 One fourth of the octahedral cloak with one segment homogenized by unit cells (left) and the cloaking performances (right). When implanted into the cloak, the unit cell was rescaled.

We choose the isotherm that passes through vertex $A_{2}$ and derived the standard deviation with a different number of unit cells. The number of cells is calculated as dividing the volume of the homogenized segment by the volume ofone cell. As shown in Fig. 17, decreasing standard deviation (i.e. increasing effectiveness) can be observed with an increasing number of cells. Interested readers can conduct more quantitative analysis towards the effectiveness of this homogenized cloak following the presented approach, but it is beyond our scope due to space limit.

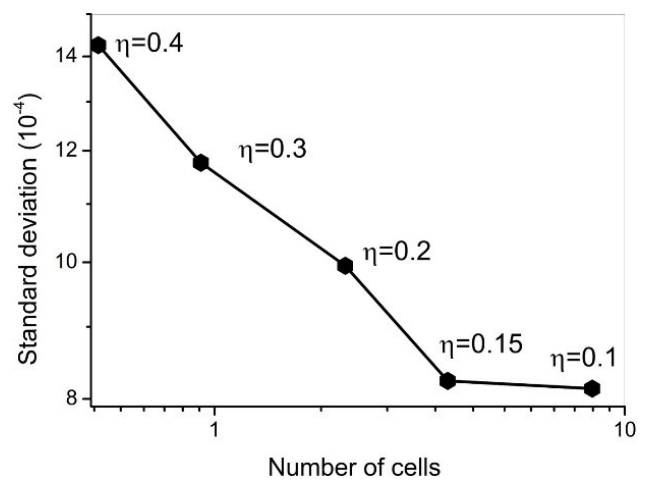

Fig. 17 Logarithm of the standard deviation for the measured isotherm versus number of elementary cells.

Three-dimensional thermal cloaks were first realized with bulk isotropic materials in Ref. [25, 26]. The bilayer strategy was then extended to the design of full-parameter omnidirectional elliptical cloaks. ${ }^{34}$ These authors advanced significantly this field. The bilayer method they proposed is direct and easy to realize with natural materials. However, we notice that in these impressive works, thermal insulators were added to create cloaking effects. Besides, only a few shapes (spherical and elliptical) were considered. In designing cloaks with complicated shapes, it would be far from trivial to obtain the correct bilayer design. In contrast, the method we propose is not constrained by the thermal insulation layer requirement. We achieve the homogenization of 3D thermal metamaterials by solving annex problems of the thermostatic type on a periodic cell, which is a direct approach that applies to arbitrary shapes. As mentioned above, there exist various effective structures. One can design the homogenized cell considering both the availability of materials and the difficulty of fabrication.

\section{Conclusion}

We have proposed a method to design three dimensional polyhedral cloaks, where the cloak is divided into tetrahedral parts. Constitutive properties are directly derived from typical coordinates by a straightforward one-step linear mapping. An octahedral cloak and a polyhedral cloak with 32 faces were designed to validate the proposed method. Simulation results show that the designed cloaks work well in cloaking objects after a certain lapse of time. As most of arbitrarily shaped cloaks can be approximated by polyhedrons, the proposed method can be applied to a wide range of geometries. As illustrative cases, we approximated spherical and cylindrical cloaks by polyhedrons and obtained the approximated material properties. It was shown that the approximated cloaks indeed 
achieve good cloaking.

The geometrical parameters used in the transformation affect the properties of each segment and thus the cloaking performance. An increment in $\eta_{1}$ provides a larger range of material properties, but at the sacrifice of some cloaking performance. In real applications, the parameters can be flexibly adjusted to achieve a trade off between cloaking performance and complexity of the material properties.

Finally, we conducted an analysis of the effectiveness of multi-layered cloaks. In the static regime, the cloak's shape directly affects convergence. However, in the transient regime convergence depends on both the lapse of time and the shape of cloaks. The cloaking performance deteriorates at short times, and becomes gradually better after a certain lapse of time. Homogenization of 3D thermal metamaterials was also investigated and it was found that $3 \mathrm{D}$ cloaks become more efficient with more built elementary cell.

\section{Conflict of Interest}

There are no conflicts to declare.

\section{Acknowledgements}

We are grateful to the support by the EIPHI Graduate School (contract "ANR-17-EURE-0002") and by the French Investissements d'Avenir program, project ISITEBFC (contract "ANR-15-IDEX-03"). Financial supports from the National Natural Science Foundation of China (11732002 and 11672089) are also gratefully acknowledged. SG is thankful for an invited professorship position in the group of Prof. Richard Craster in the mathematics department at Imperial College London (2018-2019).

\section{References}

1. J. B. Pendry, D. Schurig, and D. R. Smith, Science, 2006, 312, 1780-1782.

2. U. Leonhardt, Science, 2006, 312, 1777-1780.

3. L. Dolin, Izv. Vyssh Uchebn. Zaved. Radiofizika, 1961, 4, 964-967.

4. M. Kadic, T. Bückmann, R. Schittny, and M. Wegener, Rep. Prog. Phys., 2013, 76, 126501.

5. D. Schurig, J. Mock, B. Justice, S. A. Cummer, J. B. Pendry, A. Starr, and D. R. Smith, Science, 2006, 314, 977-980.

6. J. Li and J. B. Pendry, Phys. Rev. Lett., 2008, 101, 203901.

7. H. Chen, C. T. Chan, and P. Sheng, Nat. Mat., 2010, 9, 387-396.

8. G. W. Milton, M. Briane, and J. R. Willis, New J. Phys., 2006, 8, 248.

9. T. Bückmann, M. Thiel, M. Kadic, R. Schittny, and M. Wegener, Nat. Commun., 2014, 5, 1-6.

10. M. Farhat, S. Guenneau, and S. Enoch, Phys. Rev. Lett., 2009, 103, 024301.

11. M. Brun, S. Guenneau, and A. B. Movchan, Appl. Phys. Lett., 2009, 94, 061903.

12. T. Bückmann, M. Kadic, R. Schittny, and M. Wegener, P. Natl. A Sci., 2015, 112, 4930-4934.

13. N. Stenger, M. Wilhelm, and M. Wegener, Phys. Rev. Lett., 2012, 108, 014301.

14. M. Farhat, S. Guenneau, S. Enoch, A. Movchan, F. Zolla, and A. Nicolet, New J. Phys., 2008, 10, 115030.

15. S. A. Cummer and D. Schurig, New J. Phys., 2007, 9, 45
16. M. Farhat, S. Enoch, S. Guenneau, and A. Movchan, Phys. Rev. Lett., 2008, 101, 134501.

17. S. Guenneau and T. Puvirajesinghe, J. R. Soc. Interface., 2013, 10, 20130106.

18. R. Schittny, M. Kadic, T. Bückmann, and M. Wegener, Science, 2014, 345, 427-429.

19. R. Schittny, A. Niemeyer, M. Kadic, T. Bückmann, A. Naber, and M. Wegener, Optica, 2015, 2, 84-87.

20. M. McCall, J. B. Pendry, V. Galdi, Y. Lai, S. Horsley, J. Li, J. Zhu, R. C. Mitchell Thomas, O. Quevedo Teruel, P. Tassin, et al., J. Optics, 2018, 20, 063001 .

21. C. Fan, Y. Gao, and J. Huang, Appl. Phys. Lett., 2008, 92, 251907.

22. S. Guenneau, C. Amra, and D. Veynante, Opt. Express,2012, 20, 8207-8218.

23. R. Schittny, M. Kadic, S. Guenneau, and M. Wegener, Phys. Rev. Lett.,2013, 110, 195901.

24. S. Narayana and Y. Sato, Phys. Rev. Lett., 2012, 108, 214303

25. T. Han, X. Bai, D. Gao, J. T. Thong, B. Li, and C. W. Qiu, Phys. Rev. Lett., 2014, 112, 054302 .

26. H. Xu, X. Shi, F. Gao, H. Sun, and B. Zhang, Phys. Rev. Lett.,2014, 112, 054301 .

27. J. Shang, B. Tian, C. Jiang, and J. Huang, Appl. Phys. Lett., 2018, 113, 261902.

28. S. Yang, L. Xu, R. Wang, and J. Huang, Appl. Phys. Lett., 2017, 111, 121908.

29. T. Han, J. Zhao, T. Yuan, D. Y. Lei, B. Li, and C. W. Qiu, Energ. Environ. Sci., 2013, 6, 3537-3541.

30. T. Han, X. Bai, J. T. Thong, B. Li, and C. W. Qiu, Adv. Mater., 2014, 26, 1731-1734.

31. Y. Li, X. Shen, Z. Wu, J. Huang, Y. Chen, Y. Ni, and J. Huang, Phys. Rev. Lett., 2015, 115, 195503.

32. T. Yang, X. Bai, D. Gao, L. Wu, B. Li, J. T. Thong, and C. W. Qiu, Adv. Mater., 2015, 27, 7752-7758.

33. X. Shen, Y. Li, C. Jiang, Y. Ni, and J. Huang, Appl. Phys. Lett., 2016, 109, 031907.

34. T. Han, P. Yang, Y. Li, D. Lei, B. Li, K. Hippalgaonkar, and C. W. Qiu, Adv. Mater., 2018, 30, 1804019.

35. Y. Li, K. J. Zhu, Y. G. Peng, W. Li, T Yang, H. X. Xu, H. Chen, X. F. Zhu, S. Fan, and C. W. Qiu, Nat. Mat., 2019, 18, 48-54.

36. G. Xu, X. Zhou, and J. Zhang, Int. J. Heat Mass Transfer, 2019, 142, 118434.

37. A. Diatta, A. Nicolet, S. Guenneau, and F. Zolla, Opt. Express, 2009, 17, 13389.

38. J. Zhang, Y. Luo, H. Chen, and B.I. Wu, JOSA B, 2008, 25, 1776-1779.

39. W. X. Jiang, J. Y. Chin, Z. Li, Q. Cheng, R. Liu, and T. J. Cui, Phys. Rev. E, 2008, 77, 066607.

40. H. Ma, S. Qu, Z. Xu, and J. Wang, Opt. Express, 2008, 16, 15449-154454.

41. M. Rahm, D. Roberts, J. Pendry, and D. Smith, Opt. Express, 2008, 16, 11555-11567.

42. G. Zhu, J. Appl. Phys., 2013, 113, 163103.

43. Q. Li and J. S. Vipperman, Appl. Phys. Lett., 2014, 105, 101906.

44. A. Nicolet, F. Zolla, and S. Guenneau, Opt. Lett.,2008, 33, 1584-1586.

45. T. Yang, L. Huang, F. Chen, and W. Xu, J. Phys. D: Appl. Phys., 2013, 46, 305102.

46. T. H. Li, D. L. Zhu, F. C. Mao, M. Huang, J. J. Yang, and S. B. Li, Front. Phys., 2016, 11, 110503.

47. I. Peralta and V. D. Fachinotti, Sci. Rep., 2017, 7, 1-8.

48. X. Wang, S. Qu, X. Wu, J. Wang, Z. Xu, and H. Ma, J. Phys. D: Appl. Phys., 2010, 43, 305501

49. T. Han, C. Qiu, and X. Tang, J. Optics, 2010, 12, 095103.

50. Y. Liu, W. Guo, and T. Han, Int. J. Heat Mass Transfer, 2017, 115, 1-5.

51. G. Xu, H. Zhang, and Y. Jin, Energ. Convers. Manage., 2018, 165, 253-262.

52. G. Xu, X. Zhou, and Z. Liu, Int. Commun. Heat Mass, 2019, 108, 104337.

53. Q. Li and J. S. Vipperman, J. Appl. Phys., 2018, 124, 035103.

54. R. V. Kohn, H. Shen, M. S. Vogelius, and M. I. Weinstein, Inverse Probl., 2008, 24, 015016

55. D. Petiteau, S. Guenneau, M. Bellieud, M. Zerrad, and C. Amra, Sci. Rep., 
$2014,4,7386$

56. R. V. Craster, S. Guenneau, H. Hutridurga, and G. A. Pavliotis, Multiscale Model. Sim., 2018, 16, 1146-1176.

57. M. Pakhnin and T. Suslina, St Petersb. Math. J., 2013, 24, 949-976.

58. S. Guenneau and F. Zolla. Prog. Electromagn. Res., 2000, 27, 91-127.

59. E. Cherkaev, Inverse Probl., 2001, 17, 1203-1218.
Publisher's Note Engineered Science Publisher remains neutral with regard to jurisdictional claims in published maps and institutional affiliations. 